\title{
Study on Behavior of Breakup and Radial Migration of Droplets in a 5-stage Compressor
}

\author{
Lanxin Sun 1,3, Tao Sun², Yigang Luan² \\ ${ }^{1}$ College of Nuclear Science and Technology, Harbin Engineering University, Harbin 150001, China \\ ${ }^{2}$ College of Power and Energy Engineering, Harbin Engineering University, Harbin 150001, China \\ ${ }^{3}$ Fundamental Science on Nuclear Safety and Simulation Technology Laboratory, Harbin 150001, \\ China \\ E-mail: sunlanxin@126.com
}

KeyWords: Wet compression; Two-phase flow; Droplet breakup; radial migration of droplet; multistage compressor.

\begin{abstract}
As an economical and effective means, water injection technology is currently used to improve the gas turbine power and efficiency. Due to the relatively long residence time of water droplets in the multistage compressor, the droplets may have a large amount of migration along the radial direction in the strong centrifugal force while they will also encounter considerable breakup because of aerodynamic shear stress and impacting onto blade surface. Numerical simulation was carried out to study motion and breakup of water droplets via Eulerian-Lagrangian multiphase flow model through a 5-stage subsonic axial compressor. Simulation results show that only very small droplets can follow the gas flow well. Droplets above 30 microns in diameter will eventually become smaller because of continuously impacting and breakup. It shows also that for large droplets radial migrations along the flow direction increase obviously.
\end{abstract}

\section{Introduction}

Because of the important role in energy industry, Gas turbines have been widely used in electric power, petrochemical, natural gas transportation, railway transportation and other important industries.

Compressor is the main factor of restricting gas turbine power increase which consumes 1/2 2/3 of the gas turbine power output [1]. In addition, operating ambient temperature will also have a certain impact on the gas turbine power output, namely temperature increases every $1^{\circ} \mathrm{C}$ while gas turbine output will decrease $0.54 \% \sim 0.90 \%[2,3]$. Therefore reducing the temperature during compression process of compressor is a very effective way to improve the gas turbine power output. Water injection technology is a newly developed technique in recent years which includes mainly three kinds such as inlet spraying, interstage spraying and water injection directively into vanes [4-6]. Water injection technology uses evaporation of coolant to cool inlet or internal flow in compressor, but does not depend on the ambient temperature and humidity, which can not only improve the engine performance, but can also reduce the pollutant emission $[7,8]$. Studies now are mainly concentrated in effect of water injection on the overall performance of gas turbine plant, but seldom in the study of detailed droplet motion and breakup occurring in the flow.

Based on the CFD Technique, numerical simulation was carried out through a 5-stage subsonic axial compressor with water injection from its inlet to study motion and breakup of droplets. Structural meshing topology method is used to discretize the computational domain, $k-\varepsilon$ turbulence model and CAB droplet breakup model are chosen for simulating turbulent flow and aerodynamic shear breakup separately, and droplet impinging wall model is also established to consider droplet impacting onto the blade surface. 


\section{Numerical Method and Physical Model}

\subsection{Governing Equations and Turbuence Model}

To accurately simulate the two-phase flow process, two-way coupling effect between continuous and discrete phase must be considered. In the solution process, the two-way coupling between the two phases is implemented by solving alternately the continuous and discrete phase equations. In order to track the droplet particles moving and evaporating in the compressed, the Eulerian-Lagrangian multiphase flow model is adopted, by using Eulerian method to solve the governing equations of mixture of air and water vapor, and using Lagrangian method to solve the governing equations of discrete phase of water droplets, and through mass, momentum and energy sources to consider the two-way coupling effect between the two phases.

1) Conservation equation of the continuous phase

Conservation equation for mass, momentum and energy are written as

$$
\begin{aligned}
& \frac{\partial \rho}{\partial t}+\nabla \cdot(\rho \vec{u})=S_{\mathrm{m}} \\
& \frac{\partial}{\partial t}(\rho \vec{u})+\nabla \cdot(\rho \vec{u} \vec{u})=-\nabla p+\nabla \cdot(\overline{\bar{\tau}})+\vec{F} \\
& \frac{\partial}{\partial t}\left(\rho h_{\mathrm{t}}\right)+\nabla \cdot\left(\vec{u}\left(\rho h_{\mathrm{t}}+p\right)\right)=\nabla \cdot(\lambda \nabla T+(\overline{\bar{\tau}} \cdot \vec{u}))+\vec{u} \cdot \vec{F}+S_{\mathrm{h}}
\end{aligned}
$$

Here, specific heats of air and water vapor are defined as quartic polynomial functions of temperature.

2) Conservation equation of the dispersed phase

The forces acting on droplet particles can consider only the aerodynamic drag force, the centrifugal force and the Coriolis force, and ignore other relatively smaller ones. For this study, the equation of droplet motion can be written as:

$$
m_{\mathrm{p}} \frac{\mathrm{d} \vec{u}_{\mathrm{p}}}{\mathrm{d} t}=\vec{F}_{\mathrm{D}}+\vec{F}_{\mathrm{R}}
$$

The rate of temperature change for the particle can be obtained from the following equation:

$$
m_{\mathrm{p}} C_{\mathrm{w}} \frac{\mathrm{d} T_{\mathrm{p}}}{\mathrm{d} t}=\pi d_{\mathrm{p}} \lambda N u\left(T-T_{\mathrm{p}}\right)+\frac{\mathrm{d} m_{\mathrm{p}}}{\mathrm{d} t} h_{\mathrm{fg}}
$$

When the particle is above the boiling point, the rate of mass transfer is determined by the convective heat transfer:

$$
\frac{\mathrm{d} m_{\mathrm{p}}}{\mathrm{d} t}=-\frac{\pi d_{\mathrm{p}} \lambda N u\left(T-T_{\mathrm{p}}\right)}{h_{\mathrm{fg}}}
$$

When the particle is at below the boiling point, the rate of mass transfer is given by the formula below:

$$
\frac{\mathrm{d} m_{\mathrm{p}}}{\mathrm{d} t}=\pi d_{\mathrm{p}} \rho_{\mathrm{v}} D_{\mathrm{v}} \operatorname{Sh} \frac{M_{\mathrm{v}}}{M} \log \left(\frac{1-f_{\mathrm{p}}}{1-f}\right)
$$

Aerodynamic shear breakup of big droplets is considered by using CAB (Cascade Atomization and Breakup model) model. This model is a further development of the TAB model (Taylor Analogy Breakup model) proposed by O'Rourke and Amsden [9] and is generally adopted in liquid sprays for its robust behavior in simulating secondary breakup of droplets.

3) Turbulence model

The standard $k-\varepsilon$ turbulence model is a semi-empirical model, with good robustness and small amount of calculation. It employs the turbulent kinetic energy equation ( $k$ equation) and turbulent kinetic energy dissipation rate equation ( $\varepsilon$ equation) to close the turbulent flow governing equations.

$$
\frac{\partial(\rho k)}{\partial t}+\nabla \cdot(\rho \vec{u} k)=\nabla \cdot\left[\left(\mu+\frac{\mu_{\mathrm{t}}}{\sigma_{\mathrm{k}}}\right) \nabla k\right]+P_{\mathrm{k}}-\rho \varepsilon
$$




$$
\frac{\partial(\rho \varepsilon)}{\partial t}+\nabla \cdot(\rho \vec{u} \varepsilon)=\nabla \cdot\left[\left(\mu+\frac{\mu_{\mathrm{t}}}{\sigma_{\varepsilon}}\right) \nabla \varepsilon\right]+\frac{\varepsilon}{k}\left(C_{\varepsilon 1} P_{\mathrm{K}}-C_{\varepsilon 2} \rho \varepsilon\right)
$$

Scalable wall function is selected to solve the flow near the wall, and this method can be applied to meshes with arbitrary precision.

\subsection{Physcal Model and Mesh Topology}

A 5-stage low speed axial compressor (Figure 1) was chosen for the present study. The compressor contains totally 11 blade rows, in which the number of inlet guide vane is 54 , the number of each rotor row is 43 , and the number of each stator row is 60.Multi-block structured grid topology method was applied to discretize the computational (Figure 2). A high quality $\mathrm{H}$ grid was adopted for both inlet and outlet blocks of each passage, while an advanced J grid was utilized for the passage block with 8 layers of $\mathrm{O}$ grid around the blade. The tip clearance region was in consideration with 6 grid elements in radial direction. The computational domain of the 5-stage compressor contains a total grid of 1.5 million elements.

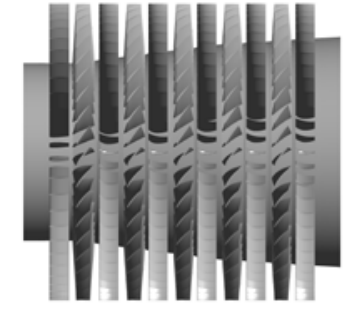

Fig. 1 Configuration of 5-stage axialcompressor

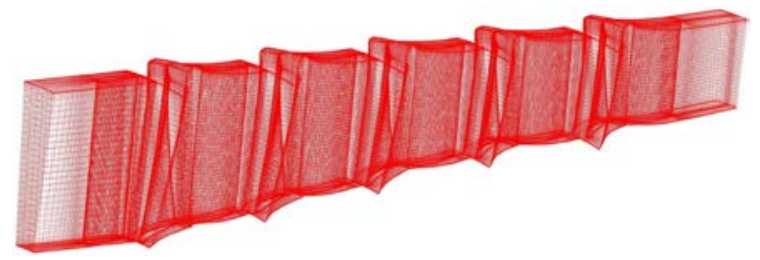

Fig. 2 Computational grid of 5-stage axial compressor

\section{Numerical Simulations and Results}

Boundary conditions of total pressure and total temperature were given at the compressor inlet as the value of $303.15 \mathrm{~K}$ and 95700 Pa respectively. Non-slip and adiabatic conditions were imposed on all the walls such as hubs, shrouds and blade surfaces. At the exit boundary, condition of static pressure or mass flowrate was given. All numerical simulations were carried out at the design point at which values for rotating speed, mass flowrate and pressure ratio are of $5561 \mathrm{r} / \mathrm{min}, 40 \mathrm{~kg} / \mathrm{s}$ and 2.05, respectively. Water is injected at a speed of $50 \mathrm{~m} / \mathrm{s}$ along the axial direction from the mid-span of compressor inlet, and the droplet diameters divided into seven groups are respectively $3,5,10$, 20, 30, 50 and $100 \mu \mathrm{m}$. When a droplet impacts onto the blade surface, breakup will happen and the size of new droplets is assumed to be determined by impacting angle. Water droplet will be captured while reaching the end wall and the trajectory tracking then finished. Droplet impingement onto blade will produce a loss of momentum, and then the restitution coefficient is assumed 0.5 for water droplets.

Figure 3 shows breakup and radial migration of water droplets of all sizes without/with considering impinging in the 5-stage compressor flow passage, and Figure 4 Figure 10 show details of breakup and radial migration of water droplets of 3, 5, 10, 20, 30, 50 and $100 \mu \mathrm{m}$ in diameter.

The calculation results show that aerodynamic shear breakup of droplets smaller than $30 \mu \mathrm{m}$, is relatively weak which indicates that the slip velocity between the two phases of water droplets and air is relatively small. However, droplets of $50 \mu \mathrm{m}$ and $100 \mu \mathrm{m}$ in diameter encounter a relatively violent aerodynamic shear breakup which shows a big slip velocity between the two phases. This leads to large droplet deformation, which reveal the unstable state of a droplet and secondary breakup occurs. For the two group droplets of $50 \mu \mathrm{m}$ and $100 \mu \mathrm{m}$, the resulting small droplets from aerodynamic shear breakup have new diameters ranged from19 to $49 \mu \mathrm{m}$ and 20 to $78 \mu \mathrm{m}$ respectively.

If the droplet/wall impinging and the resulting breakup are considered, water droplets in the 5-stage multistage compressor flow passage will encounter impinging constantly which results in a continuous breakup of big droplets and a relatively fine droplets distribution at the compressor 
outlet. It can be seen from the result that only droplets of $3 \mu \mathrm{m}$ in diameter can follow the flow well, and encounter less impinging to the blade wall than big ones which are significantly affected by impacting and breaking up. The droplets of different sizes experience continuing vigorous impacting onto the blades and breaking up, and the size of the biggest droplets is about $30 \mu \mathrm{m}$ at the compressor outlet. It indicates that considering impacting breakup, droplets larger than $30 \mu \mathrm{m}$ will eventually become smaller after constant violent breaking up, and there will no big droplets in the last stages of the compressor. It can be seen from the graph results that the smaller is the droplet the weaker is its radial migration, and migration of small droplets exhibit not obvious until the compressor exit. Big droplets show gradually increased radial migration along the flow direction, some droplets even reach the end wall.

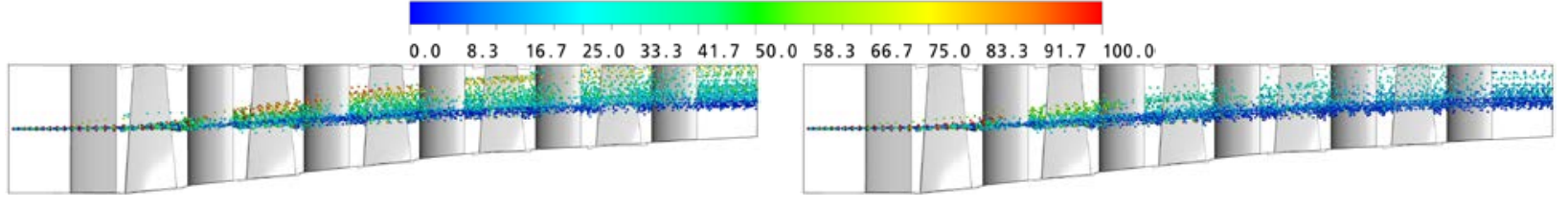

Fig. 3 Trajectories of droplets of all sizes injected from inlet mid-span

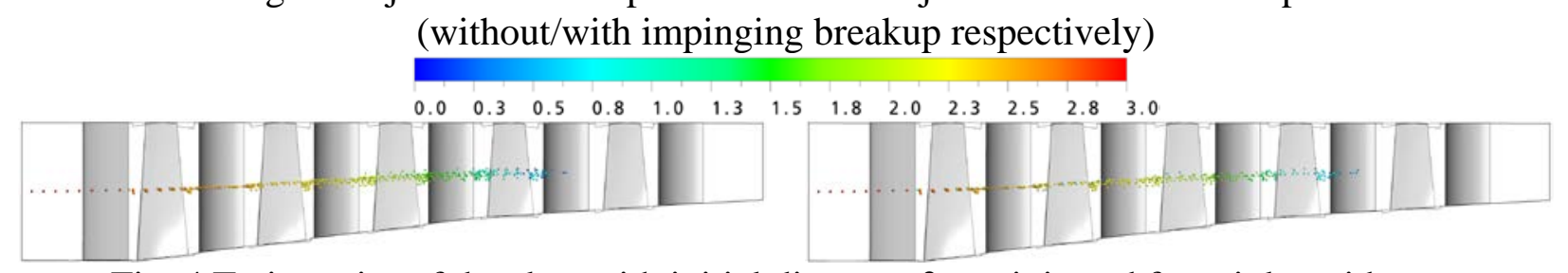

Fig. 4 Trajectories of droplets with initial diameter $3 \mu \mathrm{m}$ injected from inlet mid-span (without/with impinging breakup respectively)

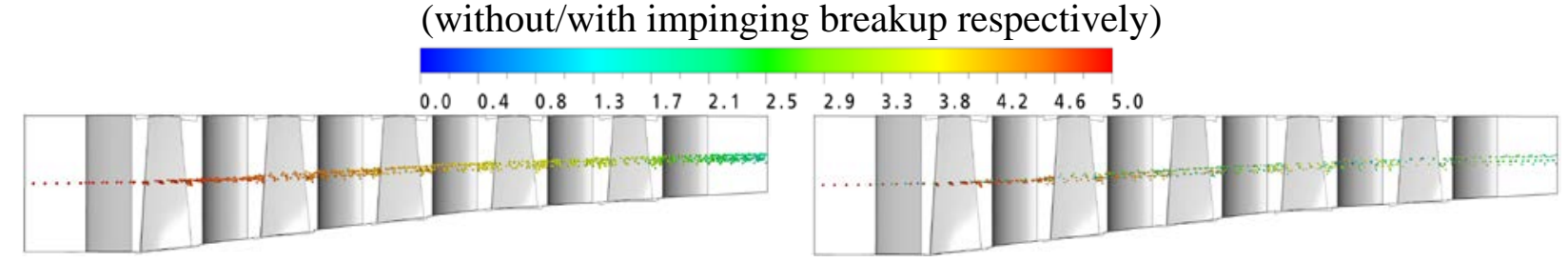

Fig. 5 Trajectories of droplets with initial diameter $5 \mu \mathrm{m}$ injected from inlet mid-span (without/with impinging breakup respectively)

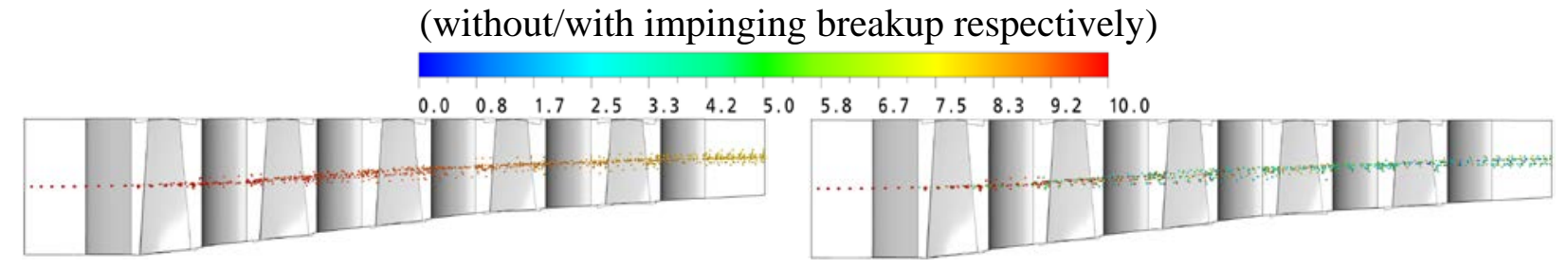

Fig. 6 Trajectories of droplets with initial diameter $10 \mu \mathrm{m}$ injected from inlet mid-span

(without/with impinging breakup respectively)

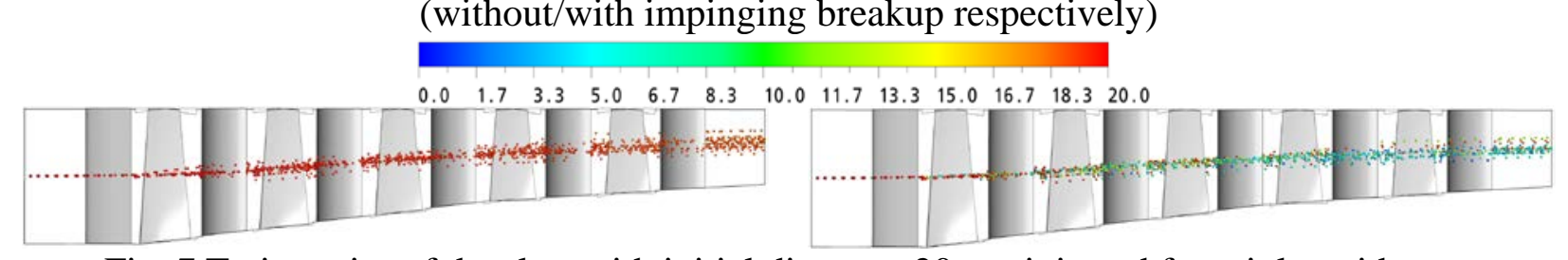

Fig. 7 Trajectories of droplets with initial diameter $20 \mu \mathrm{m}$ injected from inlet mid-span

(without/with impinging breakup respectively)

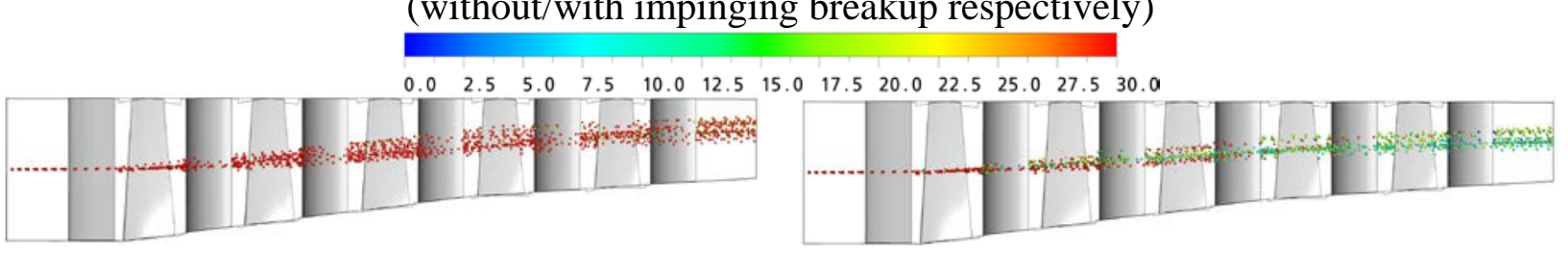

Fig. 8 Trajectories of droplets with initial diameter $30 \mu \mathrm{m}$ injected from inlet mid-span

(without/with impinging breakup respectively)

$\begin{array}{llllllllllllll}0.0 & 4.2 & 8.3 & 12.5 & 16.7 & 20.8 & 25.0 & 29.2 & 33.3 & 37.5 & 41.7 & 45.8 & 50.0\end{array}$ 

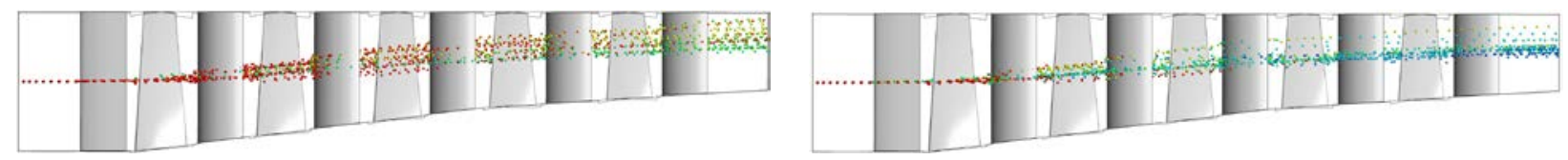

Fig. 9 Trajectories of droplets with initial diameter $50 \mu \mathrm{m}$ injected from inlet mid-span

(without/with impinging breakup respectively)

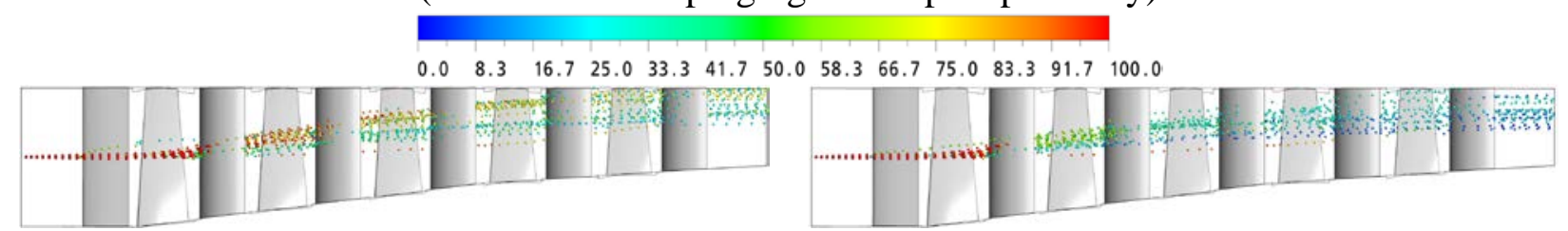

Fig. 10 Trajectories of droplets with initial diameter $100 \mu \mathrm{m}$ injected from inlet mid-span

(without/with impinging breakup respectively)

\section{Conclusions}

Numerical simulations of a 5-stage subsonic axial compressor with water injection from the inlet show the breakup and radial migration of droplets of varying diameters in the multi-stage compressor flow passage. The conclusions are as follows:

1) For droplets smaller than $30 \mu \mathrm{m}$, the slip velocity between the two phases of water droplets and air is relatively small, and they are not ready to experience aerodynamic shear breakup. However, droplets of $50 \mu \mathrm{m}$ and $100 \mu \mathrm{m}$ will inevitably encounter a relatively violent aerodynamic shear breakup resulting small droplets.

2) Water droplets in flow passage will encounter impinging constantly and result relatively fine droplets distribution at the compressor outlet. Only droplets of $3 \mu \mathrm{m}$ can follow the flow well and big droplets will experience continuing vigorous impacting onto the blades and breaking up.

3) Droplets larger than $30 \mu \mathrm{m}$ will eventually become smaller, and there will be no big droplets in the last stages of the compressor.

4) For big droplets, radial migration along the flow direction gradually increases, and some droplets even reach the end wall.

\section{Acknowledgement}

The authors would like to acknowledge the support of National Natural Science Foundation of China, Grant No. 51409067; and Harbin Engineering University Foundation HEUFN1306. Authors would also like to thank reviewers for their comments which help us improve the quality of this paper.

\section{References}

[1]. Zheng Q, Sun Y, Li S, et al. Thermodynamic analysis of wet compression process in the compressor of gas turbine[C]. Proceedings of ASME Turbo Expo 2002: Power for Land, Sea and Air, Amsterdam, Netherlands, 2002, GT-2002-30590.

[2]. Chiang Hsiaowei, Wang Paiyi. Combined cycle power augmentation by inlet fogging[C]. Proceedings of ASME Turbo Expo 2005: Power for Land, Sea and Air,

Reno-Tahoe, Nevada USA, 2005, GT-2005-68771.

[3]. Chaker M A, Meher-Homji C B. Evaporative cooling of gas turbine engines-climatic analysis and application in high humidity regions[C]. Proceedings of ASME Turbo Expo 2007: Power for Land, Sea and Air, Montreal, Canada, 2007, GT-2007-27866.

[4]. Bracco S, Pierfederici A and Trucco A, the wet compression technology for gas turbine power plants: thermodynamic model[J]. Applied Thermal Engineering, 2007, 27: 699-704.

[5]. Bhargava R K, Meher-Homji C B C B, Chaker M A, et al. Gas turbine fogging technology- 
a state of the art review: Part I-inlet evaporative fogging, analytical and experimental aspects [C]. Proceedings of ASME Turbo Expo 2005: Power for Land, Sea and Air, Reno-Tahoe, Nevada USA, 2005, GT2005-68336.

[6]. Bhargava R K, Meher-Homji C B, Chaker M A, et al. Gas turbine fogging technology-a state of the art review : Part II-overspray fogging, analytical and experimental aspects[C]. Proceedings of ASME Turbo Expo 2005: Power for Land, Sea and Air, Reno-Tahoe, Nevada USA, 2005, GT2005- 68337.

[7]. Bagnoli M, Bianchi M, Melino F, et al. A parametric study of interstage injection on GE frame 7EA gas turbine[C]. Proceedings of ASME Turbo Expo 2004: Power for Land, Sea and Air, Vienna, Austria, 2004, GT2004-53042.

[8]. Michael J. Moran, Howard N. Shapiro. Fundamentals of Engineering Thermodynamics[M]. 5th Edition. Chichester: John Wiley \& Sons Inc., 2006: 755.

[9]. Poling B E, Prausnitz J M, O’Connell J P, et al. The properties of gases and liquids[M]. New York: McGraw-Hill, 2001. 